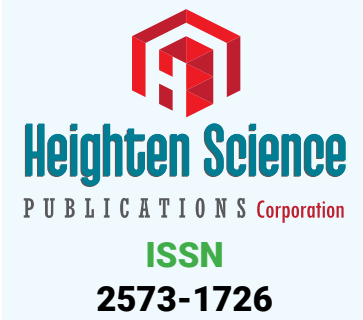

*Address for Correspondence: Gregory Ninot Montpellier Cancer Institute, 208 Avenue des Apothicaires, 34298 Montpellier, France, Tel: (+33) 4 671454 99; Email: gregory.ninot@umontpellier.fr

Submitted: 30 November 2018

Approved: 11 December 2018

Published: 12 December 2018

Copyright: (c) 2018 Maury J, et al. This is an open access article distributed under the Creative Commons Attribution License, which permits unrestricted use, distribution, and reproduction in any medium, provided the original work is properly cited

Keywords: Cancer; Physical activity; Exercise; Survival; Recurrence; Evidence

Check for updates
Review Article

\section{Role of physical activity in cancer survival and recurrence: A narrative review from relationship evidence to crucial research perspectives}

\author{
J Maury ${ }^{1}$, P Senesse ${ }^{1}$ and G Ninot ${ }^{1,2^{\star}}$ \\ ${ }^{1}$ Montpellier Cancer Institute, Montpellier, France \\ 2EA4556 Research Unit, University of Montpellier, Montpellier, France
}

\section{Abstract}

Purpose: The benefits of Physical Activity (PA) considered as a major supportive care in cancer patients, on survival, and recurrence risk is largely disseminated in public communication. However, these data must be taken with caution. The main objectives were to review the evidence and limits of studies reported regarding the post-diagnosis PA role on cancer survival and recurrence risk to secondly discuss of research perspectives on PA programs.

Method: The narrative review included all published or ongoing studies in English during the last 20 years related to PA, survival and recurrence risk with a systematic search on main databases.

Results and discussion: The current evidences regarding the PA role on survival and recurrence risk were only based on cohort studies, mainly in breast cancer. The major methodological limits identified as the lack of PA change assessment, PA level assessed largely by self-reported methods and the significant inter- but also intra- variability make the interpretation of data very. Beyond the use of rigorous RCT, the major issue is to develop adapted and personalized interventions to progressively increase PA level overtime in cancer survivors.

Conclusion: Despite the lack of causal relationship between post-diagnosis PA, survival and recurrence risk, the review underlines several interesting research perspectives. The future PA interventions, using innovative tools and integrated to the "real-life" will argued for the potential antitumoral PA role growing in literature.

\section{Introduction}

The prevalence of cancer has been steadily increasing for many years, making it the second leading global cause of death [1]. This increase is due to the optimization of diagnostic tools, main and adjuvant treatments, as well as recent developments in personalized medicine, thus promoting longer survival with cancer [2,3]. These advances justified the inclusion of supportive care during treatment and recurrence prevention periods. Physical activity (PA) is now considered as a component supportive care strategy. The role of PA interventions was to improve quality of life, more recently to decrease psychological concerns such as fatigue, anxiety, depression, pain, self-esteem trouble, body image concerns and social isolation [4]. Many cohort studies and randomized clinical trials have respectively indicated and showed benefits of PA interventions on these markers predominantly in breast cancer, but also in prostate, colon or blood cancer [5-7].

Over time, PA has also been recognized to improve specific physiological parameters. Several meta-analyses have shown strong experimental evidence to conclude that PA improve cardiorespiratory parameters [7-9], body composition and muscle mass 
$[7,8,10]$, inflammatory markers, glucose metabolism or immune system [11-13]. Given the benefits observed in the literature on these bio-psycho-social parameters, all international expert panels recommend implementation of adapted physical activity (APA) programs, as an important non-pharmacological intervention in addition to the main treatments during all care phases for most cancers and even immediately after diagnosis [14-16]. An APA program may alleviate adverse events related to cancer and its treatment, and may improve cancer treatment efficacy [17]. In fact, emerging evidence from pre-clinical studies indicate that exercise training may control cancer progression through direct effects on tumour intrinsic factors (growth rate, metastasis, tumour metabolism, and immunogenicity of the tumour) and regulates tumour growth through interplay with systemic factors [17].

Several meta-analyses and epidemiological studies seem to support this assumption $[14,18,19]$. After the cancer diagnosis, physical inactivity is raised in cancer patients due to the effect of main treatments on functional capacities that could be increased by psychological components such as anxiety, depression and reduced self-esteem [20]. Insidiously, this physical inactivity contributes not only to physiological deconditioning, psychological and social consequences of cancer described above but also to long term deleterious effects associated with increased mortality and recurrence risk, thus constituting a major public health issue. In this sense, different meta-analyses seem to report a positive association between the practice of PA after diagnosis, improvement of survival and decreased risk of recurrence [15,21-25]. These data contribute to the emerging idea that PA may be considered as a specific adjuvant therapy in cancer with a potential antitumor role [17]. Even if the benefits of PA on survival and the risk of recurrence after cancer treatment is largely disseminated and admitted in public communication, some authors highlighted numerous methodological limitations and large variability of proofs between the different cancer type suggesting that these data must be taken with caution $[14,16,23,26]$. Now regarding all these parameters, the issue well recognized in the scientific community is to conduct randomized controlled trials with rigorous methodologies to optimize the current recommendations and to define APA programs that can be integrated into the "real life" care of cancer patients.

First, the narrative review presents the evidence and the limits of published studies regarding the post diagnosis PA role on cancer survival and recurrence. Secondly, the review details research perspectives to optimize the development and evaluations of APA programs.

\section{Literature Search Method}

\section{Data sources and searches}

For this narrative review, studies published in English in the last 20 years (between January 1998 and May 2018) were identified by a systematic search on Pubmed, ScienceDirect, Web of Science and Motrial. Combinations of the following terms were used (MeSH Terms): cancer OR physical activity OR exercise OR survival OR recurrence OR tumor OR free-disease survival. The research algorithms were combined with filters to specially identify randomized controlled trials (RCT) and cohort studies. In addition, publications integrated to the present narrative review were checked from relevant meta-analysis published in each cancer type. Regarding cancer survival and recurrence parameters, we also searched the RCT in progress in ClinicalTrials.gov (U.S. National Library of Medicine), INCa database, Chinese Clinical Trial Registry (ChiCTR), Cochrane Central Register of Controlled Trials, European Clinical Trials Register, International Clinical Trials Registry Platform (ICTRP), ISRCTN registry, and UMIN Clinical Trials Registry (UMIN-CTR).

\section{Data collection process}

After screening consequently titles, abstracts and full texts, two researchers (JM, $\mathrm{PhD}$ and $\mathrm{GN}, \mathrm{PhD}$ ) independently reviewed and selected eligible publications aiming to investigate the benefits of PA on reporting outcomes (survival, recurrence). Studies were 
selected if they included adult patients with a cancer diagnosis established before the study beginning. We focused our searches on the main cancers studied in the literature: breast, lung, colon, prostate, pancreatic and hemopathy. Studies were excluded if they did not provide any details on methodology and/or PA program. Extracted data included the first author's name, year of publication, tumor stage and site, treatment status, study objective, sample size, study design and PA program methodology (intensity, frequency, modalities and duration), adherence rate and delivery mode (supervision, group, individual etc.). The results on survival, free-survival and recurrence parameters were extracted. When there was insufficient information, the relevant corresponding author was contacted.

\section{Synthesis of data}

In order to illustrate the discussion of the present narrative review, a global description of studies was firstly established to identify the current evidence available about PA effects on survival, free-survival and recurrence. For this, the number of published, in progress studies (only RCT) and meta-analysis were summarized in a graph (Figure 1). In addition, tables were established to detail the main information of published studies (Table 1) or in progress RCT (Table 2)

\section{Discussion}

An interesting but elusive role of physical activity on cancer survival and recurrence in human patients

By contrast with the well-known messages disseminate in medical literature and public health communication, the systematic review underlines clearly the lack of causal relationship between post diagnosis PA and survival or cancer recurrence.

Of the 31 studies included, only 3 are RCT in the 5 main cancers (Figure 1) [2729]. The studies incorporating experimental follow-up design reported no significant effect of a PA program on overall mortality, specific mortality or risk of recurrence. As noted by the authors, these studies were not designed or did not have the statistical power (e.g., small number of participants) to show the efficacy of the PA program on these parameters. Nevertheless, observational cohorts indicate positive relationship between PA level and overall survival (23 of 25 cohorts) or specific survival (10 of 14 cohorts) (Table 1). This relationship is described in the broad majority of studies as part of a recreational physical activity practiced daily by patients and not supervised and well-described PA program during and/or after treatments. In addition, based all studies included, 9 reported data on the risk of recurrence in cancer patients. Of these studies, only 4 reported a significant relationship between recreational PA and recurrence risk [30-33].

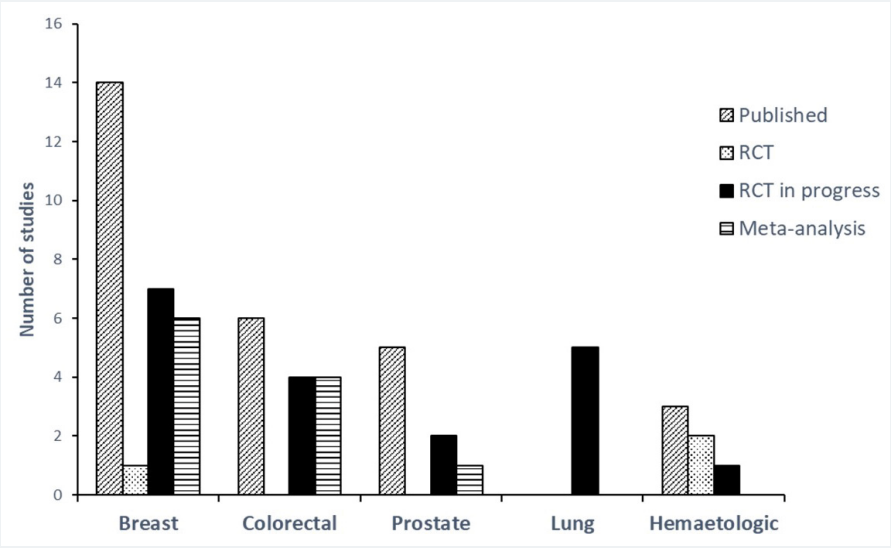

Figure 1: Number and type of studies published or in progress regarding about the relation between post diagnosis physical activity, survival and recurrence in cancer patient. 
Table 1: Characteristics of the 28 studies identified about the relation between post diagnosis physical activity, survival and recurrence in cancer patient.

\begin{tabular}{|c|c|c|c|c|c|}
\hline $\begin{array}{l}\text { Author, year, } \\
\text { country }\end{array}$ & $\begin{array}{l}\text { Cancer type, stage } \\
\text { and phase }\end{array}$ & $\begin{array}{l}\text { Study design, subjects, } \\
\text { sex }\end{array}$ & $\begin{array}{l}\text { PA measurement and } \\
\text { characteristics }\end{array}$ & Follow-up duration & Main results \\
\hline $\begin{array}{l}\text { Beasley and et al. } \\
2012 \text { (USA) [45] }\end{array}$ & $\begin{array}{c}\text { Breast cancer } \\
\text { Stages I - III } \\
\text { Post treatment ( } 18 \text { to } \\
48 \text { months after) }\end{array}$ & $\begin{array}{l}\text { Cohort (Life after Cancer } \\
\text { Epidemiology study) } \\
13302 \text { women }\end{array}$ & $\begin{array}{l}\text { Semi-quantitative questionnaires } \\
\text { (Median of } 23 \text { months post- } \\
\text { diagnosis) } \\
\text { No initial PA evaluation }\end{array}$ & $\begin{array}{l}\text { Until date of death } \\
\text { or recurrence orlast } \\
\text { contact }\end{array}$ & $\begin{array}{l}\text { Recreational PA level } \geq 10 \text { MET-hours/ } \\
\text { week associated with a } 27 \% \text { reduction in } \\
\text { all-cause of mortality and } 25 \% \text { in breast } \\
\text { cancer mortality. } \\
\text { No association with breast cancer } \\
\text { recurrence. }\end{array}$ \\
\hline $\begin{array}{l}\text { Holick and et al. } \\
2008 \text { (USA) [46] }\end{array}$ & $\begin{array}{l}\text { Breast cancer } \\
\text { All cancer stages } \\
2 \text { years after } \\
\text { diagnosis }\end{array}$ & $\begin{array}{l}\text { Cohort (Collaborative } \\
\text { Women's Longevity } \\
\text { Study) } \\
4482 \text { women }\end{array}$ & $\begin{array}{c}\text { CWLS questionnaire } \\
\text { Time spend to recreational PA } \\
\text { during the previous week } \\
\text { Assessment } 2 \text { years after } \\
\text { diagnosis } \\
\text { No initial PA evaluation }\end{array}$ & $\begin{array}{l}\text { Median follow-up : } 6 \\
\text { years }\end{array}$ & $\begin{array}{l}\text { Women who engaged in } \geq 2.8 \mathrm{MET}-\mathrm{h} / \\
\text { wk of recreational PA had a } 35 \% \text { to } 49 \% \\
\text { decreased risk of death from breast } \\
\text { cancer and all-cause of mortality. } \\
\text { No information on cancer recurrence. }\end{array}$ \\
\hline $\begin{array}{l}\text { Holmes and et al. } \\
2005 \text { (USA) [31] }\end{array}$ & $\begin{array}{l}\text { Breast cancer } \\
\text { Stage I-III ( } 30 \text { à } 55 \\
\text { years old) }\end{array}$ & $\begin{array}{l}\text { Cohort (Nurses' Health } \\
\text { study) } \\
2987 \text { women }\end{array}$ & $\begin{array}{l}\text { Leisure-time PA (questionnaire) } \\
\text { evaluated at least } 2 \text { years post- } \\
\text { diagnosis }\end{array}$ & $\begin{array}{l}\text { Median follow-up : } 8 \\
\text { years }\end{array}$ & $\begin{array}{l}\text { Women who engaged in } \geq 3 \text { MET-h/wk } \\
\text { of (dose-response effect) leisure-time } \\
\text { PA had a decreased risk of death from } \\
\text { breast cancer, all-cause of mortality and } \\
\text { recurrence in particular in women with } \\
\text { hormone responsive tumors. }\end{array}$ \\
\hline $\begin{array}{l}\text { Nechuta and et al. } \\
2016 \text { [82] }\end{array}$ & $\begin{array}{l}\text { Breast cancer } \\
\text { Stages I-III } \\
2 \text { years after } \\
\text { diagnosis }\end{array}$ & $\begin{array}{l}\text { Cohort (After Breast } \\
\text { Cancer Pooling Project) } \\
6596 \text { women estrogen } \\
\text { receptor-positive }\end{array}$ & $\begin{array}{c}\text { Self-reported information on } \\
\text { recreational PA measured on } \\
\text { average } 2,1 \text { years after diagnosis } \\
\text { No initial PA evaluation }\end{array}$ & $\begin{array}{l}\text { Median follow-up time } \\
\text { for mortality: } 12 \text { years } \\
\text { Mediand follow-up time } \\
\text { for recurrence: } 10,6\end{array}$ & $\begin{array}{c}\text { Higher level of recreational PA }(\geq 17,4 \\
\text { MET-hours/week) is inversely associated } \\
\text { with late all-cause of mortality and } 25 \% \text { in } \\
\text { breast cancer mortality. } \\
\text { No association with breast cancer } \\
\text { recurrence. }\end{array}$ \\
\hline $\begin{array}{l}\text { Irwin and et al. } \\
\quad 2011[79]\end{array}$ & $\begin{array}{l}\text { Breast cancer } \\
\text { Stages I-III }\end{array}$ & $\begin{array}{l}\text { Cohort (Women's Health } \\
\text { Initiative) } \\
4463 \text { women }\end{array}$ & $\begin{array}{c}\text { Self-reported information on } \\
\text { recreational PA measured on } \\
\text { average } 1,8 \text { years after diagnosis. } \\
\text { No initial PA evaluation }\end{array}$ & $\begin{array}{l}\text { Median follow-up time } \\
\text { for mortality: } 3,3 \text { years }\end{array}$ & $\begin{array}{l}\text { Moderate to vigourous level of } \\
\text { recreational PA ( } \geq 9 \text { MET-hours/week) is } \\
\text { inversely associated with a } 46 \% \text { reduction } \\
\text { of all-cause of mortality and } 25 \% \text { in breast } \\
\text { cancer mortality. } \\
\text { No data on breast cancer recurrence. }\end{array}$ \\
\hline $\begin{array}{l}\text { De Glas and et al. } \\
2014[76]\end{array}$ & $\begin{array}{c}\text { Breast cancer } \\
\text { All cancer stages } \\
1 \text { year after diagnosis }\end{array}$ & $\begin{array}{l}\text { Cohort (The Tamoxifen } \\
\text { Exemestane Adjuvant } \\
\text { Multicenter) } \\
435 \text { women }\end{array}$ & $\begin{array}{l}\text { PA questionnaire at } 1 \text { and } 2 \text { years } \\
\text { post diagnosis. }\end{array}$ & 2 years & $\begin{array}{l}\text { Overall survival is statically significantly } \\
\text { better for patients who were relatively } \\
\text { active. } \\
\text { No association with breast cancer- } \\
\text { specific survival and recurrence-free } \\
\text { periods. }\end{array}$ \\
\hline $\begin{array}{l}\text { Courneya and et al. } \\
2014 \text { (Canada) [27] }\end{array}$ & $\begin{array}{l}\text { Breast cancer } \\
\text { Stages I-III } \\
\text { During treatment }\end{array}$ & $\begin{array}{l}\text { Randomized controlled } \\
\text { trial (Supervised Trial } \\
\text { of Aerobic versus } \\
\text { Resistance } \\
\text { Training) } \\
242 \text { women }\end{array}$ & $\begin{array}{l}\text { AP program aerobic vs resistance } \\
\text { training) during chemotherapy ( } 3 \\
\text { sessions/week) } \\
\text { Aerobic training : } 15 \text { to } 45 \text { minutes } \\
\left.\text { on cycloergometer ( } 70 \% \mathrm{VO}_{2 \text { peak }}\right) \\
\text { Resistance training : } 9 \text { exercises } \\
\text { (8-12 repetitions) }\end{array}$ & $\begin{array}{l}8 \text { years (Median follow- } \\
\text { up: } 89 \text { months) }\end{array}$ & $\begin{array}{l}\text { No significant association between PA, } \\
\text { disease-free survival, overall survival and } \\
\text { recurrence-free interval. }\end{array}$ \\
\hline $\begin{array}{l}\text { Friedenreich and et } \\
\quad \text { al. } 2009 \text { [77] }\end{array}$ & $\begin{array}{l}\text { Breast cancer } \\
\text { All cancer stages }\end{array}$ & $\begin{array}{l}\text { Cohort (Alberta Cancer } \\
\text { Registry) } \\
1231 \text { women }\end{array}$ & $\begin{array}{c}\text { Lifetime PA Questionnaire } \\
\text { at baseline (recreational, } \\
\text { occupational and household } \\
\text { activity). } \\
\text { No PA change evaluation }\end{array}$ & $\begin{array}{l}\text { Minimal follow-up: } 8,3 \\
\text { years for recurrence } \\
\text { and } 10,3 \text { years for } \\
\text { deaths }\end{array}$ & $\begin{array}{l}\text { Highest recreational PA activity is } \\
\text { associated with a decrease in breast } \\
\text { cancer death and all deaths. } \\
\text { Moderate intensity recreational activity } \\
\text { decreased the risk of recurrence }\end{array}$ \\
\hline $\begin{array}{l}\text { Bradshaw and. } \\
\text { 2014 [73] }\end{array}$ & $\begin{array}{l}\text { Breast cancer (Long } \\
\text { Island Breast Cancer } \\
\text { Study Project) } \\
\text { All cancer stages }\end{array}$ & $\begin{array}{c}\text { Cohort } \\
1423 \text { women }\end{array}$ & $\begin{array}{l}\text { Recreational PA was assessed } \\
\text { through structured interviews at } \\
\text { baseline and follow-up using a } \\
\text { semi-questionnaire }\end{array}$ & $\begin{array}{l}\text { Median survival time } \\
\text { among women in our } \\
\text { study: } 12,7 \text { years }\end{array}$ & $\begin{array}{l}\text { Survival was improved among women } \\
\text { who were highly active after diagnosis } \\
\text { (9.0 MET } \\
\text { h/week) compared to inactive women } \\
\text { (0 MET h/week) for all-cause and breast } \\
\text { cancer-specific mortality. } \\
\text { No data on breast cancer recurrence. }\end{array}$ \\
\hline Williams, 2014 [86] & $\begin{array}{l}\text { Breast cancer } \\
\text { All cancer stages }\end{array}$ & $\begin{array}{l}\text { Cohort (National } \\
\text { Runners' and Walkers' } \\
\text { Health Surveys) } \\
986 \text { women }\end{array}$ & $\begin{array}{c}\text { PA questionnaire in order to } \\
\text { identify runner's vs walkers at } \\
\text { baseline }\end{array}$ & $\begin{array}{l}\text { Median follow-up : } 9 \\
\text { years }\end{array}$ & $\begin{array}{c}\text { For the } 986 \text { runners and walkers } \\
\text { combined, breast cancer mortality } \\
\text { decreased an average of } 23.9 \% \text { by } \\
\text { MET-H/day. There was a significantly } \\
\text { greater decrease in death risk for running } \\
\text { than walking. } \\
\text { No data on breast cancer recurrence }\end{array}$ \\
\hline $\begin{array}{l}\text { Bertram and et al. } \\
2010[42]\end{array}$ & $\begin{array}{l}\text { Breast cancer } \\
\text { Stages I-III } \\
\text { Post treatment }\end{array}$ & $\begin{array}{l}\text { Cohort (Women's } \\
\text { Healthy Eating and } \\
\text { Living) } \\
2361 \text { women }\end{array}$ & $\begin{array}{l}\text { PA questionnaire at baseline and } \\
\text { at various follow-up points }\end{array}$ & $\begin{array}{l}\text { Median follow-up : 7,1 } \\
\text { years }\end{array}$ & $\begin{array}{c}\text { Moderate to vigorous PA level at baseline } \\
\text { ( } \geq 3 \text { MET-hours/week) is associated with a } \\
53 \% \text { reduction of mortality risk. } \\
\text { Adherence to PA guidelines (10 MET-h/ } \\
\text { week) was associated with a } 35 \% \text { lower } \\
\text { mortality risk. } \\
\text { No association of PA with breast cancer } \\
\text { recurrence }\end{array}$ \\
\hline
\end{tabular}




\begin{tabular}{|c|c|c|c|c|c|}
\hline $\begin{array}{l}\text { Chen and et al. } \\
2011 \text { (China) [75] }\end{array}$ & $\begin{array}{c}\text { Breast cancer } \\
\text { Stages I-III } \\
12 \text { months following } \\
\text { diagnosis }\end{array}$ & $\begin{array}{l}\text { Cohort (Shanghai Breast } \\
\text { Cancer Survival Study) } \\
4826 \text { women }\end{array}$ & $\begin{array}{c}\text { PA level was assessed (by } \\
\text { interviews) at } 6,18 \text { and } 36 \text { months } \\
\text { postdiagnosis }\end{array}$ & $\begin{array}{l}\text { Median follow-up : 4,3 } \\
\text { years }\end{array}$ & $\begin{array}{l}\text { PA during the first } 36 \text { months } \\
\text { postdiagnosis was inversely associated } \\
\text { with total mortality and disease-specific } \\
\text { mortality }\end{array}$ \\
\hline $\begin{array}{l}\text { Sternfeld and et al. } \\
2009[84]\end{array}$ & $\begin{array}{l}\text { Breast cancer } \\
\text { Stages I-III }\end{array}$ & $\begin{array}{l}\text { Cohort (Life After Cancer } \\
\text { Epidemiology) } \\
1970 \text { women }\end{array}$ & $\begin{array}{l}\text { Self-reported frequency and } \\
\text { duration of work-related, } \\
\text { household and recreational } \\
\text { activities during the } 6 \text { months } \\
\text { prior the inclusion }\end{array}$ & $\begin{array}{l}\text { Median follow-up : 7,3 } \\
\text { years }\end{array}$ & $\begin{array}{l}\text { A statically significant protective } \\
\text { association between PA and all-cause } \\
\text { mortality. } \\
\text { No significant association between PA, } \\
\text { risk of recurrence and breast cancer } \\
\text { mortality Only a trend for higher levels ( } \geq 3 \\
\text { MET-h/week) of PA versus lowest level } \\
\text { was reported on these parameters. }\end{array}$ \\
\hline $\begin{array}{l}\text { Hellmann and et al. } \\
2010 \text { [78] }\end{array}$ & $\begin{array}{l}\text { Breast cancer } \\
\text { All cancer stages }\end{array}$ & $\begin{array}{l}\text { Cohort (Copenhagen } \\
\text { City Heart } \\
\text { Study) } \\
528 \text { women }\end{array}$ & $\begin{array}{l}\text { A self-administered questionnaire } \\
\text { and clinical } \\
\text { examination by trained personnel } \\
\text { were used to PA assessment. } \\
\text { No initial PA evaluation }\end{array}$ & $\begin{array}{l}\text { Median follow-up : 7,8 } \\
\text { years }\end{array}$ & $\begin{array}{l}\text { A moderate physical activity of } 2-4 \mathrm{~h} / \\
\text { week and a high physical activity of more } \\
\text { than } 4 \mathrm{~h} / \text { week showed no association } \\
\text { with survival after breast cancer } \\
\text { diagnosis. } \\
\text { No data on breast cancer recurrence. }\end{array}$ \\
\hline $\begin{array}{l}\text { Meyerhardt and et } \\
\text { al. } 2009 \text { [81] }\end{array}$ & $\begin{array}{l}\text { Colorectal cancer } \\
\text { nonmetastic } \\
\text { Stages I-III } \\
\text { After diagnosis }\end{array}$ & $\begin{array}{l}\text { Cohort (Health } \\
\text { Professionals Follow- } \\
\text { up Study) } \\
668 \text { men }\end{array}$ & $\begin{array}{l}\text { Leisure-time PA questionnaire } \\
\text { within } 15 \text { months after diagnosis }\end{array}$ & $\begin{array}{l}\text { Median follow-up : 8,6 } \\
\text { years }\end{array}$ & $\begin{array}{c}\text { High PA level ( } \geq 27 \text { MET-h/week) was } \\
\text { associated with improved colorectal } \\
\text { cancer-specific mortality, overall } \\
\text { mortality. }\end{array}$ \\
\hline $\begin{array}{l}\text { Baade and et al. } \\
2011[71]\end{array}$ & $\begin{array}{l}\text { Colorectal cancer } \\
\text { Stages I-III } \\
\text { After diagnosis }\end{array}$ & $\begin{array}{c}\text { Cohort } \\
1089 \text { men } \\
736 \text { women }\end{array}$ & $\begin{array}{l}\text { PA level assessed } 5 \text { months and } \\
12 \text { months after diagnosis }\end{array}$ & $\begin{array}{l}\text { Median follow-up : } 5 \\
\text { years }\end{array}$ & $\begin{array}{l}\text { Participants with some level of PA ( } \geq 150 \\
\text { minutes/week) following diagnosis } \\
\text { had } 25 \% \text { to } 28 \% \text { lower risk of all-cause } \\
\text { mortality within } 5 \text { years of diagnosis than } \\
\text { sedentary participants. Increases in PA } \\
\text { from five to } 12 \text { months postdiagnosis } \\
\text { was associated with reduced colorectal } \\
\text { specific mortality by } 32 \% \text { to } 36 \% \text { and } 31 \% \\
\text { for all-cause mortality. }\end{array}$ \\
\hline $\begin{array}{l}\text { Meyerhardt and et } \\
\text { al. 2006a [32] }\end{array}$ & $\begin{array}{l}\text { Colorectal cancer } \\
\text { Stages I-III } \\
\text { After diagnosis }\end{array}$ & $\begin{array}{l}\text { Cohort (Nurses' Health } \\
\text { study) } \\
573 \text { women }\end{array}$ & $\begin{array}{l}\text { Leisure-time PA (questionnaire) } \\
\text { evaluated at least } 1 \text { year but no } \\
\text { more than } 4 \text { years post-diagnosis }\end{array}$ & $\begin{array}{l}\text { Median follow-up : } 9,6 \\
\text { years }\end{array}$ & $\begin{array}{l}\text { High PA level ( } \geq 18 \text { MET-h/week) } \\
\text { compared with low level of PA ( } \leq 3 \text { MET-h/ } \\
\text { week) was associated with an improve } \\
\text { in colorectal cancer-specific mortality, } \\
\text { overall mortality. }\end{array}$ \\
\hline $\begin{array}{l}\text { Meyerhardt and et } \\
\text { al. 2006b [47] }\end{array}$ & $\begin{array}{l}\text { Colorectal cancer } \\
\text { Stage III } \\
\text { After diagnosis }\end{array}$ & $\begin{array}{l}\text { Cohort (Cancer and } \\
\text { Leukemia Group B) } \\
\quad 832 \text { patients }\end{array}$ & $\begin{array}{c}\text { PA level assessed by } \\
\text { questionnaire } 6 \text { months after } \\
\text { completion of adjuvant therapy }\end{array}$ & $\begin{array}{l}\text { Median follow-up : } 3,8 \\
\text { years }\end{array}$ & $\begin{array}{c}\text { High PA level ( } \geq 18 \mathrm{MET}-\mathrm{h} / \text { week) } \\
\text { compared with low level of PA ( } \leq 3 \mathrm{MET}-\mathrm{h} / \\
\text { week) was associated with a reduction } \\
\text { in recurrence-free survival and overall } \\
\text { survival. }\end{array}$ \\
\hline $\begin{array}{l}\text { Campbell and et al. } \\
2013[74]\end{array}$ & $\begin{array}{l}\text { Colorectal cancer } \\
\text { Stages I-III } \\
\text { After diagnosis }\end{array}$ & $\begin{array}{l}\text { Cohort (Cancer } \\
\text { Prevention Study) } \\
1271 \text { men } \\
991 \text { women }\end{array}$ & $\begin{array}{l}\text { Self-reported recreational and } \\
\text { leisure time PA assessed by } \\
\text { questionnaire within } 24 \text { months } \\
\text { after diagnosis }\end{array}$ & $\begin{array}{l}\text { Median follow-up : } \\
8,1 \text { years }\end{array}$ & $\begin{array}{l}\text { High recreational PA level ( } \geq 8,5 \text { MET-h/ } \\
\text { week) was associated with lower all- } \\
\text { cause mortality. } \\
\text { No data on colorectal cancer recurrence }\end{array}$ \\
\hline $\begin{array}{l}\text { Arem and et al. } \\
\quad 2015[70]\end{array}$ & $\begin{array}{l}\text { Colorectal cancer } \\
\text { All cancer stages } \\
\text { excepted stage } 0\end{array}$ & $\begin{array}{l}\text { Cohort (National } \\
\text { Institutes of Health } \\
\text { (NIH)-AARP Diet and } \\
\text { Health Study) } \\
1759 \text { patients }\end{array}$ & $\begin{array}{c}\text { Leisure time PA level assessed } \\
\text { within } 4 \text { years after cancer } \\
\text { diagnosis }\end{array}$ & $\begin{array}{l}\text { Median follow-up : } \\
11,3 \text { years }\end{array}$ & $\begin{array}{l}\text { Postdiagnosis leisure time } \mathrm{PA} \geq 7 \mathrm{~h} / \text { week, } \\
\text { compared with none, was associated with } \\
\text { a } 31 \% \text { lower all-cause mortality risk. } \\
\text { No data on colorectal cancer recurrence. }\end{array}$ \\
\hline $\begin{array}{l}\text { Wang and et al. } \\
\text { 2017[85] }\end{array}$ & $\begin{array}{l}\text { Prostate cancer } \\
\text { nonmetastatic } \\
\text { All cancer stages } \\
\text { After diagnosis }\end{array}$ & $\begin{array}{l}\text { Cohort (Cancer } \\
\text { Prevention Study } \\
\text { (CPS)-II Nutrition) } \\
5319 \text { men }\end{array}$ & $\begin{array}{l}\text { Recreational PA questionnaire at } \\
\text { least } 1 \text { year after diagnosis }\end{array}$ & $\begin{array}{l}\text { Median follow-up: } \\
10 \text { years }\end{array}$ & $\begin{array}{c}\text { High level of recreational PA ( } \geq 17.5 \text { vs } \\
3.5-<8.75 \text { MET-h/week) was associated } \\
\text { with a significant } 31 \% \text { lower risk of overall } \\
\text { prostate cancer-specific mortality. } \\
\text { No data on cancer recurrence. }\end{array}$ \\
\hline $\begin{array}{l}\text { Fridenreich and et } \\
\text { al. } 2016 \text { [77] }\end{array}$ & $\begin{array}{l}\text { Prostate cancer } \\
\text { Stages II-IV } \\
\text { After diagnosis }\end{array}$ & $\begin{array}{l}\text { Cohort (Alberta Cancer } \\
\text { Registry) } \\
830 \text { men }\end{array}$ & $\begin{array}{c}\text { Postdiagnosis PA was measured } \\
\text { up to } 3 \text { times per patient by } \\
\text { questionnaire }\end{array}$ & $\begin{array}{l}\text { Follow-up between } 1 \\
\text { and } 17 \text { years }\end{array}$ & $\begin{array}{l}\text { High level of PA ( }>119 \text { vs } \leq 42 \text { MET-h/ } \\
\text { week per year) was associated with a } \\
\text { significantly lower all-cause mortality risk. } \\
\text { No data on cancer recurrence. }\end{array}$ \\
\hline $\begin{array}{l}\text { Bonn and et al. } \\
2015 \text { [72] }\end{array}$ & $\begin{array}{l}\text { Prostate cancer } \\
\text { All cancer stages } \\
\text { After diagnosis }\end{array}$ & $\begin{array}{l}\text { Cohort (Swedish } \\
\text { National Cancer } \\
\text { Register) } \\
4623 \text { men }\end{array}$ & $\begin{array}{c}\text { Postdiagnosis PA assessed by } \\
\text { questionnaire }\end{array}$ & $\begin{array}{l}\text { Follow-up between } 1 \\
\text { and } 15 \text { years }\end{array}$ & $\begin{array}{l}\text { Statistically significant lower overall } \\
\text { mortality rates were found among men } \\
\text { engaged in } \geq 5 \text { recreational MET-h/ } \\
\text { day, walking/bicycling } \geq 20 \text { min/day, } \\
\text { performing household work } \geq 1 \mathrm{~h} / \text { day or } \\
\text { exercising } \geq 1 \mathrm{~h} / \text { wk, compared with less } \\
\text { active men within each activity type. } \\
\text { No data on cancer recurrence. }\end{array}$ \\
\hline $\begin{array}{c}\text { Kenfield and et al. } \\
2011[80]\end{array}$ & $\begin{array}{l}\text { Prostate cancer } \\
\text { nonmetastatic } \\
\text { All cancer stages } \\
\text { After diagnosis }\end{array}$ & $\begin{array}{c}\text { Cohort (Health } \\
\text { Professionals Follow-Up } \\
\text { Study) } \\
2705 \text { patients }\end{array}$ & $\begin{array}{l}\text { Self-reported leisure time PA } \\
\text { assessed every } 2 \text { years }\end{array}$ & $\begin{array}{c}\text { Follow-up between } 1 \\
\text { and } 18 \text { years }\end{array}$ & $\begin{array}{c}\text { Walked } \geq 90 \text { minutes per week at a normal } \\
\text { to very brisk pace had a } 46 \% \text { lower risk } \\
\text { of all-cause mortality compared with } \\
\text { shorter durations at an easy walking pace. } \\
\text { Men with } \geq 3 \text { hours per week of vigorous } \\
\text { activity had a } 49 \% \text { lower risk of all-cause } \\
\text { mortality. } \\
\text { No data on prostate cancer recurrence. }\end{array}$ \\
\hline
\end{tabular}




\begin{tabular}{|c|c|c|c|c|c|}
\hline $\begin{array}{l}\text { Richman and et al. } \\
2011 \text { [33] }\end{array}$ & $\begin{array}{l}\text { Prostate cancer } \\
\text { nonmetastatic } \\
\text { All cancer stages } \\
\text { After diagnosis }\end{array}$ & $\begin{array}{l}\text { Cohort (Prostate } \\
\text { Strategic Urologic } \\
\text { Research Endeavor) } \\
1455 \text { patients }\end{array}$ & PA questionnaire every 6 months & $\begin{array}{l}\text { Follow-up between } 1 \\
\text { and } 18 \text { years }\end{array}$ & $\begin{array}{l}\text { Men who walked briskly for } 3 \mathrm{~h} / \text { week } \\
\text { or more had a } 57 \% \text { lower rate of cancer } \\
\text { recurrence than men who walked at an } \\
\text { easy pace for less than } 3 \mathrm{~h} / \text { week. } \\
\text { No data on overall survival. }\end{array}$ \\
\hline $\begin{array}{l}\text { Wiskemann and et } \\
\text { al. } 2015 \text { [29] }\end{array}$ & $\begin{array}{l}\text { Allogeneic stem cell } \\
\text { transplant patients }\end{array}$ & $\begin{array}{c}\text { Randomized controlled } \\
\text { trial } \\
103 \text { patients }\end{array}$ & $\begin{array}{c}\text { Duration: } 1-4 \text { weeks prior } \\
\text { to hospital admission (non- } \\
\text { supervised); Supervised sessions } \\
\text { during hospitalisation (mean } 44 \\
\text { days) ; } 8 \text { weeks at home non- } \\
\text { supervised } \\
\text { Frequency: Combination of } \\
\text { endurance ( } 3 \text { to } 5 \text { times weekly) } \\
\text { and resistance ( } 2 \text { times weekly) } \\
\text { exercises }\end{array}$ & $\begin{array}{c}\text { Follow-up: } 2 \text { years after } \\
\text { transplatation }\end{array}$ & $\begin{array}{c}\text { Exercise intervention patients trend to } \\
\text { lower total mortality rate than controls. } \\
\text { The effect on non-relapse mortality was } \\
\text { not statiscally significant. }\end{array}$ \\
\hline $\begin{array}{l}\text { Courneya and et al. } \\
\qquad 2015 \text { [28] }\end{array}$ & $\begin{array}{l}\text { Lymphoma } \\
\text { Stages I-IV } \\
\text { During treatment }\end{array}$ & $\begin{array}{l}\text { Randomized controlled } \\
\text { trial } \\
122 \text { patients }\end{array}$ & $\begin{array}{l}\text { Duration: } 12 \text { weeks of supervised } \\
\text { aerobic exercise } \\
\text { Frequency: } 3 \text { times/week } \\
\text { Intensity: } 15 \text { to } 45 \text { minutes on } \\
\text { cycloergometer }\left(60-75 \% \mathrm{VO}_{2 \text { peak }}\right) \\
\text { PA assessment by questionnaire } \\
\text { at 6-month follow-up. }\end{array}$ & $\begin{array}{l}\text { Median follow-up : } 5,1 \\
\text { years }\end{array}$ & $\begin{array}{l}\text { No significant effect of PA program on } \\
\text { progression-free survival. } \\
\text { Specific trials designed to answer this } \\
\text { question are needed. }\end{array}$ \\
\hline $\begin{array}{l}\text { Schmid and et al. } \\
2018 \text { [83] }\end{array}$ & $\begin{array}{c}\text { First primary } \\
\text { hematologic cancer }\end{array}$ & $\begin{array}{c}\text { Cohort (Prostate } \\
\text { Strategic Urologic } \\
\text { Research Endeavor) } \\
5182 \text { patients between } \\
50 \text { and } 71 \text { years }\end{array}$ & $\begin{array}{l}\text { Leisure time PA questionnaire } \\
\text { within } 1 \text { year after diagnosis }\end{array}$ & $\begin{array}{l}\text { Median follow-up: } \\
\text { 4,4 years }\end{array}$ & $\begin{array}{l}\text { Leisure time } \mathrm{PA} \geq 4 \text { hours/week was } \\
\text { significantly associated with all-cause } \\
\text { mortality among all hematologic cancer } \\
\text { survivors, NHL survivors, myeloma } \\
\text { survivors, and leukemia survivor. } \\
\text { No data on cancer recurrence. }\end{array}$ \\
\hline
\end{tabular}

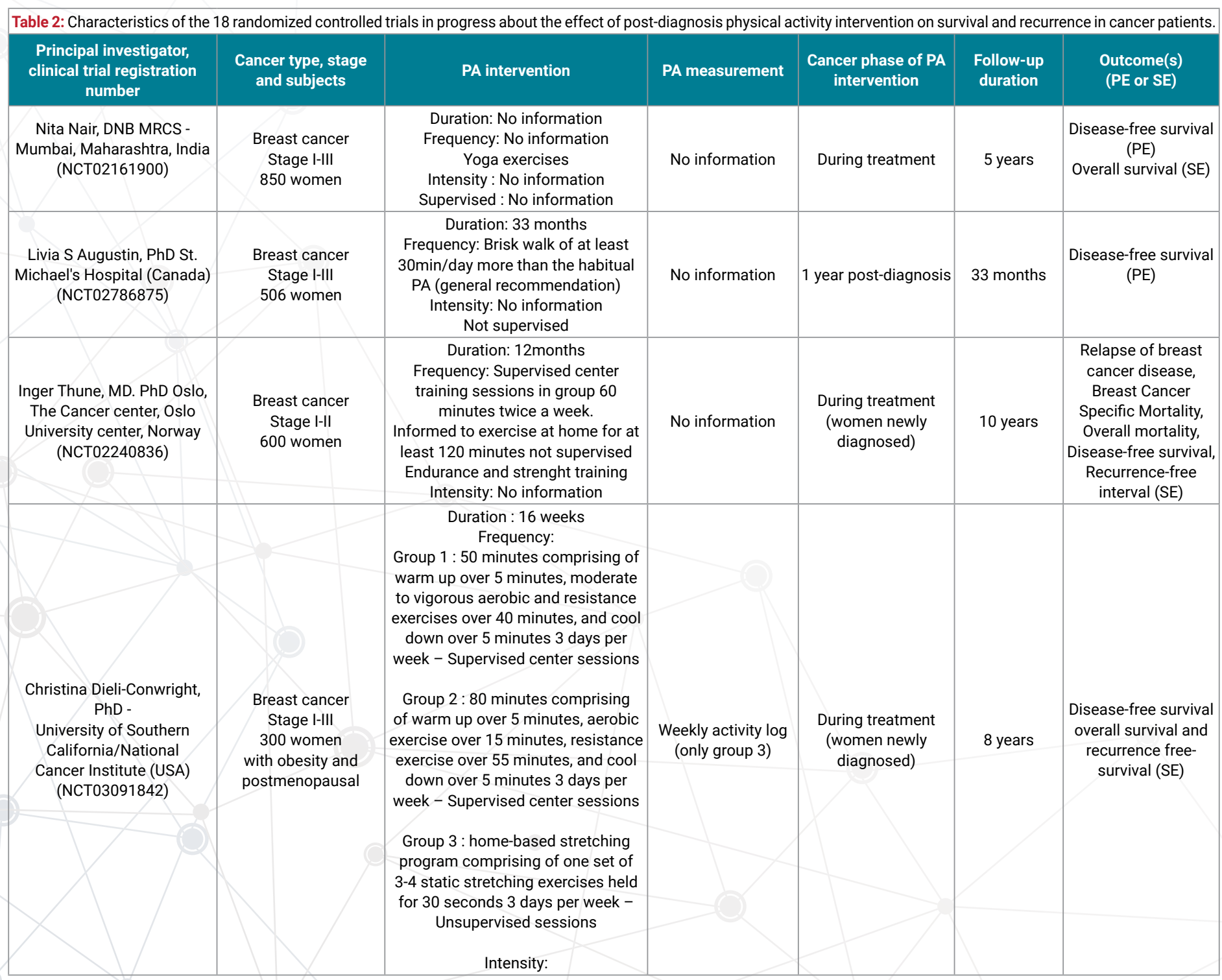




\begin{tabular}{|c|c|c|c|c|c|c|}
\hline $\begin{array}{l}\text { Pamela J Goodwin, MD, } \\
\text { MSc - } \\
\text { UHN-Mount Sinai Hospital, } \\
\text { Toronto (Canada) } \\
\text { (NCT00463489) }\end{array}$ & $\begin{array}{c}\text { Breast cancer } \\
\text { Early stage } \\
2150 \text { postmenopausal } \\
\text { women with } \\
\text { overweight }\end{array}$ & $\begin{array}{c}\text { Lifestyle intervention : } \\
\text { individual weight loss, diet and } \\
\text { physical activity goals, incorporated } \\
\text { into a 2-year standardized, } \\
\text { structured telephone and mail-based } \\
\text { intervention. } \\
\text { Recommendation : } 150-200 \text { minutes } \\
\text { per week of moderate intensity } \\
\text { aerobic PA (as walking) }\end{array}$ & $\begin{array}{c}\text { IPAQ during all } \\
\text { evaluation sessions }\end{array}$ & $\begin{array}{l}\text { During treatment } \\
\quad \text { (Letrozole) }\end{array}$ & 8 years & $\begin{array}{l}\text { Disease-free survival } \\
\quad(\mathrm{PE}) \\
\text { Overall survival (SE) }\end{array}$ \\
\hline $\begin{array}{c}\text { Jennifer Ligibel, MD - Dana- } \\
\text { Farber Cancer Institute, } \\
\text { Boston (USA) } \\
\text { (NCT02750826) }\end{array}$ & $\begin{array}{l}\text { Breast cancer } \\
\text { Early stage } \\
3136 \text { women with } \\
\text { overweight }\end{array}$ & $\begin{array}{l}\text { Telephone-based weight loss } \\
\text { intervention ( } 2 \text { years) : general } \\
\text { recommendations of PA }\end{array}$ & $\begin{array}{l}\text { Self-report of PA } \\
\text { during evaluations } \\
\text { sessions }\end{array}$ & $\begin{array}{l}12 \text { months after the } \\
\text { first diagnosis }\end{array}$ & 10 years & $\begin{array}{c}\text { Invasive disease-free } \\
\text { survival (PE), } \\
\text { Overall survival (SE), } \\
\text { Distant disease-free } \\
\text { survival }\end{array}$ \\
\hline $\begin{array}{l}\text { Antonio AGUDO, MD PhD } \\
\text { Institut Catala d'Oncologia } \\
\text { - L'Hospitalet, (Spain) } \\
\text { (NCT02035631) }\end{array}$ & $\begin{array}{l}\text { Breast cancer } \\
\text { Stage I-III } \\
2000 \text { women }\end{array}$ & $\begin{array}{l}\text { Duration : No information } \\
\text { Frequency: Two sessions per week } \\
\text { led by trained physical activity } \\
\text { monitors (unsupervised) including } \\
\text { aerobic exercise of high/moderate } \\
\text { intensity, and instruction about the } \\
\text { at-home exercise activities (3 more } \\
\text { sessions) } \\
\text { Intensity : No information }\end{array}$ & No information & $\begin{array}{l}3 \text { months post- } \\
\text { treatment and } \\
15 \text { months post- } \\
\text { diagnosis }\end{array}$ & 5 years & $\begin{array}{l}\text { Time to local and } \\
\text { distant recurrence } \\
\text { (PE), overall survival } \\
\text { (SE), Disease-free } \\
\text { survival (SE), }\end{array}$ \\
\hline $\begin{array}{l}\text { Chia-Chin Lin, PhD } \\
\text { The University of Hong Kong } \\
\text { (China) (NCT03482323) }\end{array}$ & $\begin{array}{c}\text { Lung cancer } \\
\text { Stage IIIb-IV } \\
372 \text { sedentary patients }\end{array}$ & $\begin{array}{c}\text { Duration : } 12 \text { weeks } \\
\text { Frequency : } 2 \text { sessions/week } \\
\text { supervised } \\
\text { Group } 1: \text { aerobic exercise } \\
\text { Group } 2: \text { Tai Chi intervention } \\
\text { Intensity : No information }\end{array}$ & $\begin{array}{l}\text { Accelerometer } \\
\text { before/after } \\
\text { interventions }\end{array}$ & During treatment & 1 year & $\begin{array}{l}\text { One-year survival } \\
\text { rate }(\mathrm{PE})\end{array}$ \\
\hline $\begin{array}{c}\text { Sandy Jack, PhD - } \\
\text { University Hospitals } \\
\text { Southampton NHS } \\
\text { Foundation Trust (England) } \\
\text { (NCT03334071) }\end{array}$ & $\begin{array}{l}\text { Lung cancer } \\
\text { Stage IIIb-IV } \\
100 \text { patients }\end{array}$ & $\begin{array}{c}\text { Duration : } 12 \text { weeks } \\
\text { Frequency : } 2 \text { sessions/week (1 } \\
\text { supervised in-hospital and } 1 \text { home- } \\
\text { based) } \\
\text { Intensity : Individually tailored }\end{array}$ & $\begin{array}{l}\text { Accelerometer } \\
\text { before/after } \\
\text { interventions }\end{array}$ & During treatment & 1 year & Overall survival (SE) \\
\hline $\begin{array}{c}\text { Marta Kramer Mikkelsen, } \\
\text { MHSc- } \\
\text { Rigshospitalet, (Denmark) } \\
\text { (NCT03411200) }\end{array}$ & $\begin{array}{l}\text { Lung cancer } \\
\text { locally advanced or } \\
\text { metastatic non-small } \\
\text { cell lung cancer } \\
100 \text { patients }\end{array}$ & $\begin{array}{c}\text { Duration : } 12 \text { weeks } \\
\text { Frequency : } 2 \text { sessions/week } \\
\text { Supervised and group-based } \\
\text { exercise in center two times a } \\
\text { week ( } 60 \text { minutes per session). } \\
\text { The program consists of warm-up, } \\
\text { exercises for balance and flexibility, } \\
\text { progressive resistance training, and } \\
\text { stretching and relaxation. } \\
\text { Intensity: No information }\end{array}$ & $\begin{array}{l}\text { Accelerometer } \\
\text { before/after } \\
\text { interventions }\end{array}$ & During treatment & 2 years & Overall survival (SE) \\
\hline $\begin{array}{l}\text { Joachim Wiskemann, Dr. - } \\
\text { National Center for Tumor } \\
\text { Diseases, Heideberg } \\
\text { (Germany) (NCT02055508) }\end{array}$ & $\begin{array}{l}\text { Lung cancer } \\
\text { Stage IIIb-IV } \\
232 \text { patients }\end{array}$ & $\begin{array}{c}\text { Duration : } 24 \text { weeks } \\
\text { Frequency : } 3 \text { sessions/week of } \\
\text { combined resistance and endurance } \\
\text { Program } \\
\text { Phase } 1: \text { supervised training } \\
\text { sessions } \\
\text { Phase } 2: 3 x / \text { week at least two/one } \\
\text { supervised training sessions } \\
\text { Intensity: No information }\end{array}$ & No information & During treatment & 1 year & $\begin{array}{l}\text { Overall survival, } \\
\text { progression-free } \\
\text { survival (SE) }\end{array}$ \\
\hline $\begin{array}{l}\text { Viviane Hess, Prof - } \\
\text { University Hospital, } \\
\text { Basel (Switzerland) } \\
\text { (NCT02597075) }\end{array}$ & $\begin{array}{l}\text { Colorectal cancer } \\
\text { Palliative care } \\
524 \text { patients }\end{array}$ & $\begin{array}{c}\text { Duration : } 12 \text { weeks } \\
\text { Frequency : } 2 \text { sessions/week on } \\
\text { cycloergometer in center and a } \\
\text { self-paced increase in physical } \\
\text { activity during daily life using a } \\
\text { pedometer with a daily step goal as } \\
\text { a motivational tool. } \\
\text { Intensity: No information }\end{array}$ & Podometer & During treatment & 1 year & $\begin{array}{l}\text { Progression-free } \\
\quad \text { survival (PE) } \\
\text { Overall survival (SE) }\end{array}$ \\
\hline $\begin{array}{c}\text { Onerup A., PhD - } \\
\text { Department of Surgery, } \\
\text { Institute of Clinical Sciences, } \\
\text { Sahlgrenska Academy, } \\
\text { University of Gothenburg, } \\
\text { Gothenburg (Sweden) } \\
\text { (NCT02299596) }\end{array}$ & $\begin{array}{l}\text { Colorectal cancer } \\
\text { Planned surgical } \\
\text { procedure } \\
620 \text { patients }\end{array}$ & $\begin{array}{l}\text { Duration : } 6 \text { weeks ( } 2 \text { weeks } \\
\text { before and } 4 \text { weeks after surgical } \\
\text { procedure) } \\
\text { Frequency : } 30 \text { minutes / day of } \\
\text { aerobic exercise (chosen by the } \\
\text { patient) Intensity: No information }\end{array}$ & $\begin{array}{l}\text { Questionnaire } \\
\text { (IPAQ) }\end{array}$ & $\begin{array}{l}\text { Prior and after } \\
\text { surgical procedure }\end{array}$ & 5 year & Mortality (SE) \\
\hline $\begin{array}{l}\text { Katrina A Knight, MBChB - } \\
\text { Royal Alexandra Hospita, } \\
\text { Paisley (United Kingdom) } \\
\text { (NCT03336229) }\end{array}$ & $\begin{array}{c}\text { Primary operable } \\
\text { colorectal cancer } \\
72 \text { patients }\end{array}$ & $\begin{array}{l}\text { Duration : No information } \\
\text { Frequency : No information } \\
\text { Graduated walking programme, } \\
\text { strengthening exercises and } \\
\text { respiratory muscle training (Home- } \\
\text { based telephone-guided) } \\
\text { Intensity: No information }\end{array}$ & No information & $\begin{array}{l}\text { Prior surgical } \\
\text { procedure }\end{array}$ & 3 years & Survival (SE) \\
\hline
\end{tabular}




\begin{tabular}{|c|c|c|c|c|c|c|}
\hline $\begin{array}{c}\text { Kerry Courneya, PhD - } \\
\text { University of Alberta } \\
\text { (Canada) (NCT00819208) }\end{array}$ & $\begin{array}{l}\text { Colorectal cancer } \\
\text { High risk II-III } \\
962 \text { patients } \\
\text { PA level }<150 \\
\text { minutes/week }\end{array}$ & $\begin{array}{c}\text { Duration : } 36 \text { months } \\
\text { Frequency : Phase } 1=\text { Intensive } \\
\text { intervention for } 6 \text { months } \\
\text { Phase } 2=\text { Reduced intervention for } \\
\text { months } 6-12 \\
\text { Phase } 3=\text { Minimal intervention for } \\
\text { months } 12-36 \\
\text { Intensity: Individualized from results } \\
\text { to cardiopulmonary exercise test }\end{array}$ & $\begin{array}{l}\text { Questionnaire and } \\
\text { daily record }\end{array}$ & $\begin{array}{l}\text { Post chemotherapy } \\
\text { (between } 60 \text { to } 180 \\
\text { days) }\end{array}$ & 10 years & $\begin{array}{l}\text { Disease-free survival } \\
\text { (PE) } \\
\text { Overall survival (SE) }\end{array}$ \\
\hline $\begin{array}{l}\text { Robert Newton, Dr - } \\
\text { Edith Cowan University Perth } \\
\text { (Australia) (NCT02730338) }\end{array}$ & $\begin{array}{c}\text { Prostate cancer } \\
866 \text { patients }\end{array}$ & $\begin{array}{c}\text { Duration : } 96 \text { weeks } \\
\text { Frequency: } \\
\text { First } 48 \text { weeks }=3 \text { sessions } \\
\text { supervised in an exercise clinic } \\
\text { setting with gradual transition to } \\
\text { self-management (Resistance and } \\
\text { aerobic exercises) } \\
\text { Subsequent } 48 \text { years = self } \\
\text { managed exercise with one visit at } \\
\text { the beginning of each cycle } \\
\text { Intensity: High intensity }\end{array}$ & Questionnaire & During treatment & 3 years & $\begin{array}{c}\text { Overall survival (PE) } \\
\text { Disease progression } \\
\text { (SE) }\end{array}$ \\
\hline $\begin{array}{l}\text { Miklos Pless, Prof. - } \\
\text { Kantonsspital Winterthur } \\
\text { KSW (Switzerland) } \\
\text { (NCT02585362) }\end{array}$ & $\begin{array}{c}\text { Advanced prostate } \\
\text { cancer } \\
88 \text { patients }\end{array}$ & $\begin{array}{c}\text { Duration : } 12 \text { weeks } \\
\text { Frequency : } 2 \text { sessions/week in } \\
\text { center and } 1 \text { home-based session/ } \\
\text { week } \\
\text { Intensity: No information }\end{array}$ & No information & During treatment & 6 months & Overall survival (SE) \\
\hline $\begin{array}{c}\text { Jarden M., PhD - } \\
\text { Copenhagen University } \\
\text { Hospital (Denmark) } \\
\text { (NCT01404520) }\end{array}$ & $\begin{array}{l}70 \text { Patients with } \\
\text { leukemia }\end{array}$ & $\begin{array}{c}\text { Duration : } 12 \text { weeks } \\
\text { Frequency : } 3 \text { hour/week supervised } \\
\text { in-hospital program of aerobic } \\
\text { (stationary cycle) and functional } \\
\text { muscle training at high intensity. } \\
\text { Combined with relaxation training } \\
\text { (low intensity) and unsupervised in- } \\
\text { home walking ( } 3 \text { sessions/week) }\end{array}$ & $\begin{array}{c}\text { Podometer, } \\
\text { questionnaire and } \\
\text { logbook }\end{array}$ & During treatment & 1 year & Overall survival (SE) \\
\hline
\end{tabular}

All these data issue from literature are supported by 11 meta-analyses [21,2325,34-40]. It should be noted that all the studies included in meta-analyses are found in our literature review without exception. In these works, the positive relationship between PA level, overall and specific mortality is described only in breast, colon and prostate cancers with increased effects in patients with higher PA levels. For example, the meta-analysis of Schmid et al. [24,] reported a $41 \%$ decrease in overall mortality and a $34 \%$ decrease in specific mortality in breast cancer patients with physical activity levels $\geq 3$ MET.h/week. The authors also describe a dose-response effect with decreases of $13 \%$ and $34 \%$ in overall mortality for increases of 5 MET.h/week and 15 MET.h/week respectively. Similar results have been found in colon and prostate cancers $[21,24,35$,]. Interestingly, although these meta-analyses did not suggest a causal relationship, the positive effect of PA on overall or specific survival was observed after statistical analyses incorporating adjustment on different clinically relevant variables in cancer (e.g., cancer stage, treatment, smoking status, co-morbidities etc.). Regarding the relationship between the PA level and the risk of recurrence, only meta-analyses performed in breast cancer reported relevant data even if the authors specified that the results must be taken with caution given the small number of studies included in the analysis [21,24,25,34,]. Thus, Ibrahim and Al-Homaidh 2011 reported a $24 \%$ decreased risk of recurrence in breast cancer patients with AP levels $\geq 3$ MET.h/week.

However, as mentioned in some meta-analyses and systematic reviews [16,21,41,], this narrative review highlights major limitations to be considered in datainterpretation from the literature (Table 1).

1/ Change in PA level during post-diagnosis follow-up of patients has rarely been studied in observational cohorts with baseline assessment present in less than $20 \%$ of studies [36]. To our knowledge, only one study cohort was interested in the effect of the change in PA level, evaluated 1 year after the baseline assessment, on overall survival but reported no significant relationship [42]. Yet the change in PA level during follow-up is a major consideration in assessing the effect of AP level on survival. For example, it has recently been shown that an increase in PA levels assessed before and after cancer diagnosis was associated with a reduction in long-term mortality [24]. 
2/ In all cohorts, PA was assessed with declarative methods using long-term recall questionnaires or interviews. This methodology is interesting for epidemiological studies to estimate individual PA dose, but with important risks of bias [43]. Some authors argued that these self-report methods capture only $50 \%$ of objectively measured energy expenditure [44]. In this sense, Matthews et al. [43] recommend to combine these questionnaires with objective measure tools such as accelerometer or pedometer. Another alternative should be to repeat frequently self-report during patient follow-up estimating the PA level achieved in short term recall to reduce the risk of bias [43].

3/ The review also points out heterogeneity of cancer and stage follow by observational cohorts. For example, no cohort has been achieved in lung cancer which remains the highest mortality rate cancer (Figure 1). More than $50 \%$ of the studies included breast cancer with a large severity, stage I, II or III. These data therefore indicate that it is scientifically inconsistent to overgeneralize the causal relationship between the PA level, overall and specific survival to all cancer types.

4/ The review also underlines the heterogeneity between the beginning and the end (from 0 to 4 years after diagnosis of PA programs, as well as the duration of followup (from 1 to 18 years after diagnosis) (Table 1 ).

5/ Finally, the review indicates substantial differences between PA programs. For example, the cut-offs defined can vary from 3 to 27 MET.h/week depending on the studies and cancer types [45-47]. Most studies use the term of "recreational PA" associated with survival or risk of recurrence. This term includes a broad range of activities such as running, swimming, cycling, gardening, and skiing (etc.) that patients can practice daily without having a rigorous PA program. It is well recognized that an inadequate description of PA in studies is a major factor contributing to the absence of PA prescription by clinicians $[48,49]$.

All these limitations make the analysis and interpretation of these data very complex even after rigorous meta-analyses published. For these reasons, the role of PA and PA supervised programs in survival and recurrence risk remains elusive in patients with cancer as has been suggested by some researchers $[16,21,23,41$,$] .$

\section{Major challenges of research perspectives on PA, survival and recurrence}

As it is well recognized in literature, physical inactivity, defined as an PA level of less than 150 minutes per week by several academic organizations [50-52] is a major problem in cancer survivors [14,20]. Although data are heterogeneous across studies, the percentage of inactive patients increased compared to healthy population $[51,53,54]$. Because of their important side effects, the main antitumor treatments favor this phenomenon in this population [14]. For example, a large French cohort within multiple cancer types showed that about $90 \%$ of patients do not increase their level of PA two years after diagnosis and this observation is worsen at 5 years [55]. Thus, the major issue for future research projects is to develop adapted programs to increase the PA level in cancer survivors for regular and self-administered long-term practice, which is essential to improve survival and reduce recurrence risk [56].

The first message widely found in the literature is to carry out rigorous RCT with appropriate designs and well-described PA program. Indeed, it seems essential to evaluate non-pharmacological interventions such as PA programs [48,49]. Overall, the analysis of 137 clinical trials evaluating non-pharmacological interventions showed that $61 \%$ did not report sufficient details to be replicated [26,57]. These methodological considerations are necessary to guide physicians and researchers towards the implementation of structured and personalized AP programs, provided that the characteristics of the populations studied are homogenized as much as possible [58]. 
However, when we read the declaration of RCT in progress on ClinicalTrials.gov, methodological limitations persist. As shown in table 2, only 7 out of the 18 ongoing RCT were specifically designed to investigate the survival or cancer recurrence as primary endpoint. For trials analyzing survival or cancer recurrence as a secondary endpoint, we will therefore find limitations in the interpretation of the data, particularly in relation to the statistical power problem described in numerous RCT already published [27-29]. In this case, it seems important to standardize, at least by cancer type, the parameters evaluated (e.g., duration of follow-up to assess survival) as well as the tools to measure accurately PA in patients as discussed previously [43]. It should also be noted that only one RCT in progress combines two tools to determine PA dose and five use an objective measure such as accelerometer or pedometer (Table 2). This standardization will be easier to compare studies and for meta-analyses [58]. In the same sense, we mentioned the importance of evaluating the change of PA level over the duration of patient follow-up and this has been implemented in four ongoing RCT (Table 2). From a methodological viewpoint, it is also necessary to define precisely the three major components of PA programs, duration, frequency and intensity $[14,48,49]$. Most of the data available on RCT in progress do not allow to precisely identify the content and intensity of the PA program sessions, although the duration and frequency are generally well described (Table 2).

Beyond these methodological considerations, the content of PA programs should lead to long-term behavior change in cancer survivors. As reported by Hudis and Jones [59], promoting long-term PA is a truly complex task, particularly in cancer. Although the global objective would be for all patients to achieve at least the general recommendations (150 minutes/week of moderate to vigorous PA combined with 2-3 sessions/week of resistance training), it is well recognized that minimal changes in PA levels in inactive patients would produce significant improvements in survival and recurrence risk $[14,20]$. Yet low adherence to PA interventions associated with inclusion difficulties is frequently reported in clinical trials both during and after the treatment phase $[26,60]$. Thus, the major currently question is: what tools have been identified in the literature to increase the PA level at long-term in cancer survivors? [26].

From this PA behavior change perspective, taking into account barriers and facilitators to practice is one of the major components [20,61-63]. To date, the main barriers reported in literature differ according to the cancer type, in particular regarding physiological health disorders, even some components seem transverse to all patients: lack of time, geographical location in relation to the hospital center, difficulty of access to practice. However, the broad majority of studies on the PA effects in cancer survivors have been conducted in hospital centers, which may explain the adhesion and inclusion difficulties described [26,60]. As shown in table 2, although many ongoing RCT are being conducted in hospital centers (2/3 of studies), there is growing interest in implementing PA programs integrated into the care pathway based on the facilitators recommended. The two main components to facilitate the practice of long-term regular PA in cancer survivors are home-based interventions and supervised sessions $[26,61,64-66]$. Indeed, the number of studies evaluating the effects of using new technologies (e.g. telephone, e-mail, web/online support, mobile application etc.) to promote PA among cancer patients continues to increase $[64,65]$. According to some authors, the evolution of traditional model follow-up in cancer patient centers is necessary and must be reconfigured to correspond to the real needs of patients [65]. To this notion of home-based intervention is added the notion of supervised session. To our knowledge, all the studies carried out or in progress (Table 2) on the implementation and evaluation of home-based programs to induce a PA behavior change in cancer survivors are conducted unsupervised, whether by telephone, e-mail, web support or via mobile applications. The use of these non-face-to-face new technologies, defined as "broad-reaching approaches", is a financial and feasible interesting solution, but limited 
and heterogeneous effects on long-term PA levels are reported [64,67]. As reported by Buffart et al. [56], the effects are greater when programs used professional supervision, whether on quality of life, physical ability or patient adherence to the intervention. An important point raised by the authors is that PA sessions should be conducted by a qualified health professional, whether supervised or not $[56,65]$.

A potentially more effective solution evocated in literature to encourage patients towards autonomy in PA practice would be to combine supervised sessions in hospitalcenter with unsupervised home-based sessions, even if the risks of low adherence to the supervised program remain [26]. Currently, 7 ongoing RCT (Table 2) have used this methodology and the results will allow us to make progress on this point in the coming years. Regarding the intervention durations implemented, whether in published trials or in RCT in progress, we find a significant heterogeneity (from 4 weeks to 2 years) which does not allow us to determine an optimal methodology. Some authors suggest that the 12-month PA program would be too long and would not allow patients to be autonomous in their practice but studies are still needed to show this [26]. In any case, it is important that interventions respect progressiveness in the development of PA interventions. This progressiveness must apply both to the intensity of the sessions but also to the frequency. The implementation of AP programs with a pyramidal design with (1) progressive then degressive evolution of the number of supervised sessions per week associated with (2) an increase of the sessions number in autonomy during follow-up, constitutes an interesting solution to be evaluated scientifically to induce a PA behavior change in cancer survivors. Another innovative solution mentioned in the literature but, to our knowledge, not yet evaluated in cancer patients is the use of videoconferencing, which would make it possible to carry out PA interventions both at home and supervised by a qualified health professional. Another research perspective to be developed would be to integrate PA interventions into comprehensive patient support as recommended in the latest report of the World Cancer Res Fund International. The AP programs would then be combined with adapted nutritional follow-up and education sessions to potentially optimize benefits on survival. For example, disease management program would sensitize the patient to the interest and benefits of PA, on physical abilities, quality of life and survival, which are facilitating components highlighted in the literature $[20,62,63]$. For all these solutions, future studies on PA integrated in the care pathway should be directed towards a clinical application favoring the implementation of an adapted and personalized medicine directly involving the patient.

From an ethical perspective, many issues need to be addressed in future clinical research. According to some studies, patients would prefer to begin PA interventions after treatment, but this is linked to a lack of patient information and awareness, which further justifies education sessions [20,62]. However, the cancer diagnosis would constitute a "teachable moment" to PA behavior change, even more given the benefits described in the literature regarding PA interventions carried out during treatment $[63,68,69]$. Another important point is to target the less active patients because of the specific needs of this population and yet it would appear that they are the least involved in clinical trials (inclusion bias) [41]. Finally, the question of setting up a control group is also open to discussion because today it seems difficult to not purpose PA interventions to cancer patients given the current general recommendations. Thus, in future clinical research, it would be appropriate to carry out several groups of interventions by varying different tools and/or modalities: supervised $v s$ unsupervised sessions; place of practice (hospital vs home-based); duration, frequency and intensity of the intervention; individual vs group sessions; follow-up tools (videoconferencing, mobile application, telephone follow-up, etc.).

\section{Conclusion}

The PA role on survival and cancer recurrence is based only on observational cohort in the five major solid tumors studied: breast, colorectal, lung, prostate and hematology. 
The lack of RCT showing causal relationship and the methodological limitations of cohort makes data interpretation complex to generalize. Nevertheless, these observational cohort studies provide many interesting research issues to investigate the specific PA role on survival and recurrence risk. The major challenge of current and future research projects is to develop adapted and personalized PA interventions, tailored as much as possible to the type of cancer, to induce progressively a regular and self-administered long-term practice. For this purpose, it seems essential to develop RCT with rigorous methodologies and adapted PA programs that consider patients "real life" problems. Many innovative solutions exist such as mobile applications, internet support or videoconferencing, but it is now necessary to find PA interventions that induce optimal benefits, using home-based and/or supervised sessions depending on the populations studied, on the PA level, survival and the recurrence risk. These observations and clinical research hypotheses also suggest the potential antitumoral PA role reported in pre-clinical animal studies [17]. Although the underlying mechanisms are still largely unknown in different forms of cancer, some parameters such as inflammation, the immune system or insulin appear to be involved in tumor development. These potentially PA-modulated parameters could also be incorporated into the evaluation of future clinical research projects.

\section{Acknowledgment}

The authors gratefully acknowledge the Supportive Care Department team and Research Department team (Institut du Cancer de Montpellier, ICM), the Fondation ARC, the SIRIC Montpellier Cancer (Grant INCa-DGOS-Inserm 6045), and Cedric Baudinet (V@Si company).

\section{References}

1. Global Burden of Disease Cancer Collaboration, Fitzmaurice C1, Allen C2, Barber RM2, Barregard L. et al. Global, Regional, and National Cancer Incidence, Mortality, Years of Life Lost, Years Lived With Disability, and Disability-Adjusted Life-years for 32 Cancer Groups, 1990 to 2015: A Systematic Analysis for the Global Burden of Disease Study. JAMA Oncol. 2017; 3: 524-528. Ref.: https://goo.gl/qt1GT9

2. Lowy DR, Collins FS. Aiming High - Changing the Trajectory for Cancer. N Engl J Med. 2016; 374: 1901-1904. Ref.: https://goo.gl/iom4XT

3. Siegel RL, Miller KD, Jemal A. Cancer statistics, 2018: Cancer Statistics, 2018. CA Cancer J Clin. 2018; 68: 7-30. Ref.: https://goo.gl/ocDvFd

4. Courneya KS, Friedenreich $\mathrm{CM}$. Physical exercise and quality of life following cancer diagnosis: $\mathrm{A}$ literature review. Ann Behav Med. 1999; 21: 171-179. Ref.: https://goo.gl/apsLpN

5. Cramp F, Daniel J. Exercise for the management of cancer-related fatigue in adults. Cochrane Database Syst Rev. 2008; CD006145. Ref.: https://goo.gl/DasDop

6. Mishra SI, Scherer RW, Snyder C, Geigle PM, Berlanstein DR, et al. Exercise interventions on healthrelated quality of life for people with cancer during active treatment. Clinical Otolaryngology. 2012; CD008465. Ref.: https://goo.gl/DGLyUi

7. Speck RM, Courneya KS, Mâsse LC, Duval S, Schmitz KH. An update of controlled physical activity trials in cancer survivors: a systematic review and meta-analysis. J Cancer Surviv. 2010; 4: 87-100. Ref.: https://goo.gl/KU9xPT

8. Fong DY, Ho JW, Hui BP, Lee AM, Macfarlane DJ, et al. Physical activity for cancer survivors: metaanalysis of randomised controlled trials. BMJ. 2012; 344: e70-e70. Ref.: https://goo.gl/wFfLdU

9. Jones LW, Liang Y, Pituskin EN, Battaglini CL, Scott JM, et al. Effect of Exercise Training on Peak Oxygen Consumption in Patients with Cancer: A Meta-Analysis. Oncologist. 2011; 16: 112-120. Ref.: https://goo.gl/qHWiPM

10. Strasser B, Steindorf K, Wiskemann J, Ulrich CM. Impact of Resistance Training in Cancer Survivors: A Meta-Analysis. Med Sci Sports Exerc. 2013; 45: 2080-2090. Ref.: https://goo.gl/ZdKaSX

11. Ballard-Barbash R, Friedenreich CM, Courneya KS, Siddiqi SM, McTiernan A, et al. Physical Activity, Biomarkers, and Disease Outcomes in Cancer Survivors: A Systematic Review. J Natl Cancer Inst. 2012; 104: 815-840. Ref.: https://goo.gl/Y6kkfW 
12. Hojman P. Exercise protects from cancer through regulation of immune function and inflammation. Biochem Soc Trans. 2017; 45: 905-911. Ref.: https://goo.gl/KfXfQT

13. Löf M1, Bergström K, Weiderpass E. Physical activity and biomarkers in breast cancer survivors: A systematic review. Maturitas. 2012; 73: 134-142. Ref.: https://goo.gl/BeQVRA

14. Ancellin R, Gaillot-de Saintignon J. Bénéfices de l'activité physique pendant et après cancer : des connaissances scientifiques aux repères pratiques. Oncologie. 2017; 19: 95-107. Ref.: https://goo.gl/Njr7mx

15. Kohler LN, Garcia DO, Harris RB, Oren E, Roe DJ, et al. Adherence to Diet and Physical Activity Cancer Prevention Guidelines and Cancer Outcomes: A Systematic Review. Cancer Epidemiol Biomarkers Prev. 2016; 25: 1018-1028. Ref.: https://goo.gl/sKFPCE

16. Stout NL, Baima J, Swisher AK, Winters-Stone KM, Welsh J. A Systematic Review of Exercise Systematic Reviews in the Cancer Literature (2005-2017). PM R. 2017; 9: S347-S384 Ref.: https://goo.gl/JYc7P3

17. Hojman P, Gehl J, Christensen JF, Pedersen BK. Molecular Mechanisms Linking Exercise to Cancer Prevention and Treatment. Cell Metab. 2018; 27: 10-21. Ref.: https://goo.gl/RQcngm

18. Moore SC, Lee IM, Weiderpass E, Campbell PT, Sampson JN, et al. Association of Leisure-Time Physical Activity With Risk of 26 Types of Cancer in 1.44 Million Adults. JAMA Intern Med. 2016; 176 816. Ref.: https://goo.gl/NRjCwU

19. Rezende LFM, Sá TH, Markozannes G, Rey-López JP, Lee IM, et al. Physical activity and cancer: an umbrella review of the literature including 22 major anatomical sites and 770000 cancer cases. $\mathrm{Br} \mathrm{J}$ Sports Med. 2017; 52: 826-833. Ref.: https://goo.gl/xiJCb9

20. Ormel HL, van der Schoot GGF, Sluiter WJ, Jalving M, Gietema JA, et al. Predictors of adherence to exercise interventions during and after cancer treatment: A systematic review. Psychooncology. 2018; 27: 713-724. Ref.: https://goo.gl/248swz

21. Benke IN, Leitzmann MF, Behrens G, Schmid D. Physical activity in relation to risk of prostate cancer: a systematic review and meta-analysis. Ann Oncol. 2018; 29: 1154-1179. Ref.: https://goo.gl/Vf37Zn

22. Kerr J, Anderson C, Lippman SM. Physical activity, sedentary behaviour, diet, and cancer: an update and emerging new evidence. The Lancet Oncol. 2017; 18: e457-e471. Ref.: https://goo.gl/KYe7ch

23. Lahart IM, Metsios GS, Nevill AM, Carmichael AR. Physical activity for women with breast cancer after adjuvant therapy. Cochrane Database Syst Rev. 2018; CD011292. Ref.: https://goo.gl/p6tHhv

24. Schmid D, Leitzmann MF. Association between physical activity and mortality among breast cancer and colorectal cancer survivors: a systematic review and meta-analysis. Ann Oncol. 2014; 25: $1293-$ 1311. Ref.: https://goo.gl/MvWPb7

25. Wu W, Guo F, Ye J, Li Y, Shi D. Pre- and post-diagnosis physical activity is associated with survival benefits of colorectal cancer patients: a systematic review and meta-analysis. Oncotarget. 2016; 7: 52095-52103. Ref.: https://goo.gl/VrtrqL

26. Bourke L, Homer KE, Thaha MA, Steed L, Rosario DJ, et al. Interventions to improve exercise behaviour in sedentary people living with and beyond cancer: a systematic review. $\mathrm{Br} J$ Cancer. 2014; 110: 831-841. Ref.: https://goo.gl/pmF7Kt

27. Courneya KS, Segal RJ, McKenzie DC, Dong H, Gelmon K, et al. Effects of Exercise during Adjuvant Chemotherapy on Breast Cancer Outcomes: Med Sci Sports Exerc. 2014; 46: 1744-1751. Ref.: https://goo.gl/5iSzPu

28. Courneya KS, Friedenreich CM, Franco-Villalobos C, Crawford J4, Chua N, et al. Effects of supervised exercise on progression-free survival in lymphoma patients: an exploratory follow-up of the HELP Trial. Cancer Causes \& Control. 2015; 26: 269-276. Ref.: https://goo.gl/ipGiWr

29. Wiskemann J, Kleindienst N, Kuehl R, Dreger P, Schwerdtfeger R, et al. Effects of physical exercise on survival after allogeneic stem cell transplantation: Effects of exercise on survival after transplant. Int J Cancer. 2015; 137: 2749-2756. Ref.: https://goo.gl/2KCGaV

30. Friedenreich CM, Gregory J, Kopciuk KA, Mackey JR, Courneya KS. Prospective cohort study of lifetime physical activity and breast cancer survival. Int J Cancer. 2009; 124: 1954-1962. Ref.: https://goo.gl/ABV2hp

31. Holmes MD, Chen WY, Feskanich D, Kroenke CH, Colditz GA. Physical Activity and Survival after Breast Cancer Diagnosis. JAMA. 2005; 293: 2479-2486. Ref.: https://goo.gl/hNEXdM

32. Meyerhardt JA, Giovannucci EL, Holmes MD, Chan AT, Chan JA, et al. Physical Activity and Survival after Colorectal Cancer Diagnosis. J Clin Oncol. 2006; 24: 3527-3534. Ref.: https://goo.gl/moCiYp 
33. Richman EL, Kenfield SA, Stampfer MJ, Paciorek A, Carroll PR, et al. Physical Activity after Diagnosis and Risk of Prostate Cancer Progression: Data from the Cancer of the Prostate Strategic Urologic Research Endeavor. Cancer Res. 2011; 71: 3889-3895. Ref.: https://goo.gl/bRNhgr

34. Ibrahim EM, Al-Homaidh A. Physical activity and survival after breast cancer diagnosis: metaanalysis of published studies. Med Oncol. 2011; 28: 753-765. Ref.: https://goo.gl/PYZjca

35. Je Y, Jeon JY, Giovannucci EL, Meyerhardt JA. Association between physical activity and mortality in colorectal cancer: A meta-analysis of prospective cohort studies: Physical activity and colorectal cancer mortality. Int J Cancer. 2013; 133: 1905-1913. Ref.: https://goo.gl/wV2LLZ

36. Lahart IM, Metsios GS, Nevill AM, Carmichael AR. Physical activity, risk of death and recurrence in breast cancer survivors: A systematic review and meta-analysis of epidemiological studies. Acta Oncologica. 2015; 54: 635-654. Ref.: https://goo.gl/FqTDsT

37. Lee CHA, Kong JC, Ismail H, Riedel B, Heriot A. Systematic Review and Meta-analysis of Objective Assessment of Physical Fitness in Patients Undergoing Colorectal Cancer Surgery. Dis Colon Rectum. 2018; 1. Ref.: https://goo.gl/Z2uZ5u

38. Otto SJ, Korfage IJ, Polinder S, van der Heide A, de Vries E, et al. Association of change in physical activity and body weight with quality of life and mortality in colorectal cancer: a systematic review and meta-analysis. Support Care Cancer. 2015; 23: 1237-1250. Ref.: https://goo.gl/4S2RhS

39. Soares Falcetta F, de Araújo Vianna Träsel H, de Almeida FK, Rangel Ribeiro Falcetta M, Falavigna $M$, et al. Effects of physical exercise after treatment of early breast cancer: systematic review and meta-analysis. Breast Cancer Res Treat. 2018; 170: 455-476. Ref.: https://goo.gl/VNMdoX

40. Zhong S, Jiang T, Ma T, Zhang $X$, Tang J, et al. Association between physical activity and mortality in breast cancer: a meta-analysis of cohort studies. Eur J Epidemiol. 2014; 29: 391-404. Ref.: https://goo.gl/zccZaa

41. Cormie P, Zopf EM, Zhang X, Schmitz KH. The Impact of Exercise on Cancer Mortality, Recurrence, and Treatment-Related Adverse Effects. Epidemiol Rev. 2017; 39: 71-92. Ref.: https://goo.gl/STh3Vb

42. Bertram LA, Stefanick ML, Saquib N, Natarajan L, Patterson RE, et al. Physical activity, additional breast cancer events, and mortality among early-stage breast cancer survivors: findings from the WHEL Study. Cancer Causes Control. 2011; 22: 427-435. Ref.: https://goo.gl/tA5f4N

43. Matthews CE, Moore SC, George SM, Sampson J, Bowles HR. Improving Self-reports of Active and Sedentary Behaviors in Large Epidemiologic Studies. Exerc Sport Sci Rev. 2012; 40: 118-126. Ref.: https://goo.gl/ZHpMWa

44. Neilson HK, Robson PJ, Friedenreich CM, Csizmadi I. Estimating activity energy expenditure: how valid are physical activity questionnaires? Am J Clin Nutr. 2008; 87: 279-291. Ref.: https://goo.gl/wkxx7h

45. Beasley JM, Kwan ML, Chen WY, Weltzien EK, Kroenke $\mathrm{CH}$, et al. Meeting the physical activity guidelines and survival after breast cancer: findings from the after breast cancer pooling project. Breast Cancer Res Treat. 2012; 131: 637-643. Ref.: https://goo.gl/DQNaqk

46. Holick CN, Newcomb PA, Trentham-Dietz A, Titus-Ernstoff L, Bersch AJ, et al. Physical Activity and Survival after Diagnosis of Invasive Breast Cancer. Cancer Epidemiology Biomarkers \& Prevention. 2008; 17: 379-386. Ref.: https://goo.gl/Cx6zyr

47. Meyerhardt JA, Heseltine D, Niedzwiecki D, Hollis D, Saltz LB, et al. Impact of Physical Activity on Cancer Recurrence and Survival in Patients With Stage III Colon Cancer: Findings From CALGB 89803. J Clin Oncol. 2006; 24: 3535-3541. Ref.: https://goo.gl/sSGf5J

48. Hoffmann TC, Maher CG, Briffa T, Sherrington C, Bennell K, et al. Prescribing exercise interventions for patients with chronic conditions. CMAJ. 2016; 188: 510-518. Ref.: https://goo.gl/cR2MNz

49. Persson G, Brorsson A, Ekvall Hansson E, Troein M, Strandberg EL. Physical activity on prescription (PAP) from the general practitioner's perspective - a qualitative study. BMC Fam Pract. 2013; 14: Ref.: https://goo.gl/7XXgEf

50. Rock CL, Doyle C, Demark-Wahnefried W, Meyerhardt J, Courneya KS, et al. (2012). Nutrition and physical activity guidelines for cancer survivors. CA Cancer J Clin. 2012; 62: 242-274. Ref.: https://goo.gl/WuSLJ7

51. Schmitz KH, Courneya KS, Matthews C, Demark-Wahnefried W, Galvão DA, et al. American College of Sports Medicine Roundtable on Exercise Guidelines for Cancer Survivors. Med Sci Sports Exerc. 2010; 42: 1409-1426. Ref.: https://goo.gl/Wk8p4S

52. Sedentary Behaviour Research Network. Letter to the Editor: Standardized use of the terms "sedentary" and "sedentary behaviours." Appl Physiol Nutr Metab. 2012; 37: 540-542. Ref.: https://goo.gl/5RsutP 
53. Coups EJ, Ostroff JS. A population-based estimate of the prevalence of behavioral risk factors among adult cancer survivors and noncancer controls. Prev Med. 2005; 40: 702-711. Ref.: https://goo.gl/qBH9jG

54. Mowls DS, Brame LS, Martinez SA, Beebe LA. Lifestyle behaviors among US cancer survivors. J Cancer Surviv. 2016; 10: 692-698. Ref.: https://goo.gl/rXAN2e

55. La vie cinq ans après un diagnostic de cancer. 2018; 364. Ref.: https://goo.gl/p1xTbh

56. Buffart LM, Galvão DA, Brug J, Chinapaw MJ, Newton RU. Evidence-based physical activity guidelines for cancer survivors: Current guidelines, knowledge gaps and future research directions. Cancer Treat Rev. 2014; 40: 327-340. Ref.: https://goo.gl/1oepk9

57. Hoffmann TC, Erueti C, Glasziou PP. Poor description of non-pharmacological interventions: analysis of consecutive sample of randomized trials. BMJ. 2013; 347: f3755-f3755. Ref.: https://goo.gl/9TYXJK

58. Jones LW, Alfano CM. Exercise-oncology research: Past, present, and future. Acta Oncologica. 2013; 52: 195-215. Ref.: https://goo.gl/YKn8Lf

59. Hudis $\mathrm{CA}$, Jones L. Promoting exercise after a cancer diagnosis: easier said than done. $\mathrm{Br} \mathrm{J}$ Cancer 2014; 110: 829-830. Ref.: https://goo.gl/qqqupY

60. Courneya KS, Segal RJ, Gelmon K, Reid RD, Mackey JR, et al. Predictors of Supervised Exercise Adherence during Breast Cancer Chemotherapy. Med Sci Sports Exerc. 2008; 40: 1180-1187. Ref.: https://goo.gl/tVt4aM

61. Bluethmann SM, Vernon SW, Gabriel KP, Murphy CC, Bartholomew LK. Taking the next step: a systematic review and meta-analysis of physical activity and behavior change interventions in recent post-treatment breast cancer survivors. Breast Cancer Res Treat. 2015; 149: 331-342. Ref.: https://goo.gl/AkLHxd

62. Clifford BK, Mizrahi D, Sandler CX, Barry BK, Simar D, et al. Barriers and facilitators of exercise experienced by cancer survivors: a mixed methods systematic review. Support Care Cancer. 2018; 26: 685-700. Ref.: https://goo.gl/Z3XDMS

63. Demark-Wahnefried W, Rogers LQ, Alfano CM, Thomson CA, Courneya KS, et al. Practical clinical interventions for diet, physical activity, and weight control in cancer survivors: Clinical Interventions for Weight Loss, Diet, and Physical Activity. CA Cancer J Clin. 2015; 65: 167-189. Ref.: https://goo.gl/y6nnwC

64. Goode AD, Lawler SP, Brakenridge CL, Reeves MM, Eakin EG. Telephone, print, and Web-based interventions for physical activity, diet, and weight control among cancer survivors: a systematic review. J Cancer Surviv. 2015; 9: 660-682. Ref.: https://goo.gl/F62f7t

65. Groen WG, van Harten WH, Vallance JK. Systematic review and meta-analysis of distance-based physical activity interventions for cancer survivors (2013-2018): We still haven't found what we're looking for. Cancer Treat Rev. 2018; 69: 188-203. Ref.: https://goo.gl/nRRu78

66. Wong JN, McAuley E, Trinh L. Physical activity programming and counseling preferences among cancer survivors: a systematic review. Int J Behav Nutr Phys Act. 2018; 15: Ref.: https://goo.gl/N9JLYu

67. Eakin EG, Hayes SC, Haas MR, Reeves MM, Vardy JL, et al. Healthy Living after Cancer: a dissemination and implementation study evaluating a telephone-delivered healthy lifestyle program for cancer survivors. BMC Cancer. 2015; 15: Ref.: https://goo.gl/NrHyVZ

68. Liss MA, White M, Natarajan L, Parsons JK. Exercise Decreases and Smoking Increases Bladder Cancer Mortality. Clin Genitourin Cancer. 2017; 15: 391-395. Ref.: https://goo.gl/h2XyVr

69. Spence RR, Heesch KC, Brown WJ. Colorectal cancer survivors' exercise experiences and preferences: qualitative findings from an exercise rehabilitation programme immediately after chemotherapy: Exercise rehabilitation study: qualitative findings. Eur J Cancer Care (Engl). 2011; 20: 257-266. Ref.: https://goo.gl/TCfN2o

70. Arem H, Pfeiffer RM, Engels EA, Alfano CM, Hollenbeck A, et al. Pre- and Postdiagnosis Physical Activity, Television Viewing, and Mortality Among Patients With Colorectal Cancer in the National Institutes of Health-AARP Diet and Health Study. J Clin Oncol. 2015; 33: 180-188. Ref.: https://goo.gl/inrcpK

71. Baade PD, Meng X, Youl PH, Aitken JF, Dunn J, et al. The Impact of Body Mass Index and Physical Activity on Mortality among Patients with Colorectal Cancer in Queensland, Australia. Cancer Epidemiol Biomarkers Prev. 2011; 20: 1410-1420. Ref.: https://goo.gl/4Ds5Uh

72. Bonn SE, Sjölander A, Lagerros YT, Wiklund F, Stattin P, et al. Physical Activity and Survival among Men Diagnosed with Prostate Cancer. Cancer Epidemiol Biomarkers Prev. 2015; 24: 57-64. Ref.: https://goo.gl/EUsoRh 
73. Bradshaw PT, Ibrahim JG, Khankari N, Cleveland RJ, Abrahamson PE, et al. Post-diagnosis physical activity and survival after breast cancer diagnosis: the Long Island Breast Cancer Study. Breast Cancer Res Treat. 2014; 145: 735-742. Ref.: https://goo.gl/8WobnQ

74. Campbell PT, Patel AV, Newton CC, Jacobs EJ, Gapstur SM. Associations of Recreational Physical Activity and Leisure Time Spent Sitting with Colorectal Cancer Survival. J Clin Oncol. 2013; 31: 876885. Ref.: https://goo.gl/uuiKYz

75. Chen X, Lu W, Zheng W, Gu K, Matthews CE, et al. Exercise after Diagnosis of Breast Cancer in Association with Survival. Cancer Prev Res (Phila). 2011; 4: 1409-1418. Ref.: https://goo.gl/Rf25Tv

76. de Glas NA, Fontein DB, Bastiaannet E, Pijpe A, De Craen AJ, et al. Physical activity and survival of postmenopausal, hormone receptor-positive breast cancer patients: Results of the Tamoxifen Exemestane Adjuvant Multicenter Lifestyle study: Physical Activity in Breast Cancer. Cancer. 2014; 120: 2847-2854. Ref.: https://goo.gl/5ZcUsJ

77. Friedenreich CM, Wang Q, Neilson HK, Kopciuk KA, McGregor SE, et al. Physical Activity and Survival After Prostate Cancer. Eur Urol. 2016; 70: 576-585. Ref.: https://goo.gl/2YE3u8

78. Hellmann SS, Thygesen LC, Tolstrup JS, Grønbaek M. Modifiable risk factors and survival in women diagnosed with primary breast cancer: results from a prospective cohort study. Eur J Cancer Prev. 2010; 19: 366-373. Ref.: https://goo.gl/xXVNqg

79. Irwin ML, McTiernan A, Manson JE, Thomson CA, Sternfeld B. et al. Physical Activity and Survival in Postmenopausal Women with Breast Cancer: Results from the Women's Health Initiative. Irwin ML1, McTiernan A, Manson JE, Thomson CA, Sternfeld B. 2011; 4: 522-529. Ref.: https://goo.gl/U38mPY

80. Kenfield SA, Stampfer MJ, Giovannucci E, Chan JM. Physical Activity and Survival After Prostate Cancer Diagnosis in the Health Professionals Follow-Up Study. J Clin Oncol. 2011; 29: 726-732. Ref.: https://goo.gl/r326eN

81. Meyerhardt JA, Giovannucci EL, Ogino S, Kirkner GJ, Chan AT. et al. Physical Activity and Male Colorectal Cancer Survival. Arch Intern Med. 2011; 169: 2102. Ref.: https://goo.gl/jd9tba

82. Nechuta S, Chen WY, Cai H, Poole EM, Kwan ML, et al. A pooled analysis of post-diagnosis lifestyle factors in association with late estrogen-receptor-positive breast cancer prognosis. Int $\mathrm{J}$ Cancer. 2016; 138: 2088-2097. https://goo.gl/yXc1QL

83. Schmid D, Behrens G, Arem H, Hart C, Herr W, et al. Pre- and post-diagnosis physical activity, television viewing, and mortality among hematologic cancer survivors. PLOS ONE. 2018; 13: e0192078. Ref.: https://goo.gl/PCD8uC

84. Sternfeld B, Weltzien E, Quesenberry CP Jr, Castillo AL, Kwan M, et al. Physical Activity and Risk of Recurrence and Mortality in Breast Cancer Survivors: Findings from the LACE Study. Cancer Epidemiol Biomarkers Prev. 2009; 18: 87-95. Ref.: https://goo.gl/4eVv2B

85. Wang Y, Jacobs EJ, Gapstur SM, Maliniak ML, Gansler T, et al. Recreational Physical Activity in Relation to Prostate Cancer-specific Mortality Among Men with Nonmetastatic Prostate Cancer. Eur Urol. 2017; 72: 931-939. Ref.: https://goo.gl/nL1kD7

86. Williams PT. Significantly greater reduction in breast cancer mortality from post-diagnosis running than walking: Post-diagnosis exercise vs breast cancer mortality. Int J Cancer. 2014; 135: 11951202. Ref.: https://goo.gl/zRmgNj 